\title{
Alcoholic liver disease has more bleeding tendency during liver transplant operation
}

\author{
Mun Chae CHOI, Dong Jin JOO*
}

Department of Surgery, Yonsei University College of Medicine, Seoul, Korea

Introduction: Alcohol liver disease (ALD) includes a wide clinical spectrum from acute alcoholic hepatitis to severe cirrhosis and/or hepatocellular carcinoma. Until now, there was no report to reveal the bleeding tendency of ALD compared to other diseases in liver transplantation (LT). Thus, we analyzed the blood loss and transfusion amount during operation according to the etiologies of liver disease and model for end stage liver disease (MELD) score.

Methods: Out of 874 recipients who underwent LT, a total of 146 patients were excluded by our exclusion criteria. We compared 728 recipient's baseline characteristics, operation time, blood loss, and transfusion amounts between ALD and non-ALD according to MELD score.

Results: The number of patients in ALD groups was 130 (17.9\%), and 598 (82.1\%) in non-ALD group. ALD group showed younger age, higher MELD score, and more proportion of deceased donor liver transplantation than non-ALD group. Overall blood loss, RBC, FFP, Platelets transfusion of ALD group were significantly higher than non-ALD group. When we divided the patients into two group according to the MELD score 20, ALD group showed significant blood loss, RBC, and FFP transfusion in both higher and lower MELD groups even though there were no significant differences of INR and PLT counts between two groups in the higher MELD subgroup. Conclusions: ALD showed more bleeding tendency than non-ALD during LT operation. These findings imply that transplant surgeon need to prepare for blood loss during operation for ALD patients regardless of the MELD score. 